\title{
Controllability of a Nonhomogeneous String and Ring under Time Dependent Tension
}

\author{
S. A. Avdonin ${ }^{1}$ * B. P. Belinskiy ${ }^{2}$ and L. Pandolfi ${ }^{3}$ \\ ${ }^{1}$ University of Alaska Fairbanks, Fairbanks, AK 99775-6660, USA \\ ${ }^{2}$ University of Tennessee at Chattanooga, 615 McCallie Avenue, Chattanooga, TN 37403-2598, USA \\ ${ }^{3}$ Politecnico di Torino, Corso Duca degli Abruzzi 24, 10129 Torino, Italy
}

\begin{abstract}
We study controllability for a nonhomogeneous string and ring under an axial stretching tension that varies with time. We consider the boundary control for a string and distributed control for a ring. For a string, we are looking for a control $f(t) \in L^{2}(0, T)$ that drives the state solution to rest. We show that for a ring, two forces are required to achieve controllability. The controllability problem is reduced to a moment problem for the control. We describe the set of initial data which may be driven to rest by the control. The proof is based on an auxiliary basis property result.
\end{abstract}

Key words: string equation, ring equation, exact controllability, Riesz basis, moment problem AMS subject classification: 93B05, 35C20, 35L20, 93C20

\section{Introduction}

We consider the following model that is well-known in Mechanics [21]. Let a flexible elastic string be fixed/free at the end points and be under the action of a (given) axial stretching tension that varies in time. We say that the string is controllable if, by suitable manipulation of the input (the force applied at one of the end points), the output can be made to behave in some desirable way, for example, the string goes to rest. In other words, we study boundary controllability of this system. The paper represents a continuation of the previous papers by first two authors [1], [2], and [4] and the papers [24]-[26] of the third author. In the papers [1], [4], the density of the string and ring correspondingly is constant but the tension is supposed to be the sum of a constant

${ }^{*}$ Corresponding author. E-mail: ffsaa@uaf.edu 
positive tension and a small tension that varies "slowly enough" with time; in the paper [2], the density of the string depends on the coordinate but the tension depends on time. To prove our results here (and in [1], [2], [4]), we apply the method of moments. This has been widely used in control theory of distributed parameter systems since the classical papers of H. O. Fattorini and D. L. Russell in the late 60s to early 70s (see the excellent survey [28] and book [5] for the history of the subject and complete references.) The method is based on properties of exponential families (usually in the space $L^{2}(0, T)$ ), the most important of which for control theory are minimality, the Riesz basis property and also the $\mathcal{L}$-basis property. The latter is defined to be a Riesz basis in the closure of the linear span of the family. Recent investigations into new classes of distributed systems such as hybrid systems and damped systems as well as problems of simultaneous control have raised a number of new and difficult problems in the theory of exponential families (see, e.g. [6]- [10], [12], [17]) including the theory of vector-valued exponentials [5] and many other interesting topics.

At this moment, it is important to mention that the solution to the control problem for several models described by the wave equation with the help of reduction to the moment problem is given in [27], [28], [5]-[10], [3], [12], etc. We note that in all aforementioned models, the coefficients of the partial differential equations are time independent.

The results of this paper and the previous papers [1], [2], [4], and [24]-[26] may be considered as a generalization of the classical results by D. L. Russell [27] for the one-dimensional wave equation.

The main difference between our problem of control and the previous problems is that the coefficient of the wave equation (the tension in our model) is a function of time.

We now discuss the consequences of this change in the structure of the model.

The bases encountered when the tension $P$ is constant (and in fact in most of the applications presented so far) are bases of exponential functions. If instead $P=P(t)$ varies with time, the bases to be studied are bases of functions which solve suitable differential equations; but the expression of the solutions cannot be written in closed form (see [1], [4].) This is the new difficulty encountered in the study of systems with the time-varying tension. A similar situation occurs in the study of controllability for viscoelastic materials, or equivalently, study of heat equation with memory (see [24]-[26]; we say more about these papers later.)

We note that the study of one-dimensional systems with the variable tension is not of purely academic interest. In fact, it is an important subject in itself, addressed in treatises on vibrations (see, e.g. [16], [21], and [32].) The reason is that in practice the system we are controlling is never isolated. Rather, it is a part of a train of mechanisms which transmit to the system both the controlling forces and unwanted forces which act as perturbations. In some situations these perturbations are known, since they are due to the known motion of the mechanisms, for example to the rotation of shafts.

The wave equation with the time dependent tension is studied in [1], [4]. The proof of Riesz basis property of a family of the time-dependent functions in [1] and [4] is based on the assumption that the tension varies "slowly enough" with time. This restriction is removed in [2] but only controllability of the displacement or the velocity (not both of them as usual) is proved. To the best of our knowledge, the papers [1], [2], [4], and the current paper represent the first attempt to apply 
the method of moments to equations with time dependent coefficients. As we said already, the new difficulty in this case is the absence of an explicit representation for a basis. This fact, which substantially complicates the analysis of controllability, is common to the study of controllability for heat equations with memory, i.e.

$$
y_{t}(x, t)-\gamma \triangle y(x, t)-\int_{0}^{t} a(t-s) \triangle y(x, s) d s=m(x) u(x, t),
$$

$(x, t) \in \Omega \times(0, T)$, subject to the appropriate initial and boundary conditions. Here $\Omega$ is a bounded region in $R^{n}, \gamma \geq 0$, and $m(x)$ is the characteristic function of an open subset of $\Omega$. This problem was studied first in [11] under some restrictions on the memory kernel $a(t)$ (removed in [34, 23].) Controllability is proved in these papers, but they do not suggest a constructive method of finding a control which steers a given initial condition to hit a prescribed target. (To be fair, a constructive method of finding control is mostly studied in one-dimensional case, see [1]-[10], [12], [17], [24][27], and the literature cited therein.)

The paper [24] (as well as [25]-[26]) is especially important to us. The one-dimensional version of Eq-n (1.1) with $\gamma=0$ is considered there. Controllability problem is reduced to a moment problem with respect to a system of functions. Techniques developed in $[24,26]$ allow proving that the aforementioned system is a Riesz system in spite of the fact that an explicit expression for the elements of the basis is not available. That allows solving controllability problem in a constructive way.

The main goal of this paper, concerning the wave equation with time varying coefficient $P(t)$ is to remove the restriction on the tension (used in the papers $[1,2,3,4]$ ) with the help of an idea suggested in [24]-[26].

Now we describe the equation we are going to study.

The transverse displacement of the string $y(x, t)$ satisfies the wave equation

$$
\rho y_{t t}=\left(P y_{x}\right)_{x}, 0<x<l, t>0 .
$$

We will not discuss the well-known derivation of it (see [18], [21], [32]) and only remind the basic assumptions that clarify the statement of the problem. (a) The displacement vector is perpendicular to the $x$-axis where the string is located in the equilibrium position, so that the displacements may be described by only one function $y(x, t)$. (b) The string has no resistance to bending, and hence the direction of the tension is tangential to the string. (c) The string satisfies Hooke's law. (d) $y_{x}^{2}<<1$. (e) The linear density of the string $\rho$ is a function of coordinate $x$. It follows from (b)(d) that the horizontal component $P(t)$ of the tension is independent on the coordinate $x$. Further, $P(t) y_{x}(0, t)$ represents an external vertical force applied at the end point $x=0$ of the string. We are going to study a string whose right end is fixed while the left end is subject to a vertically directed controlling force, $f(t)$. So, the initial boundary value problem (IBVP) that describes the small transverse oscillations of the string consists of the hyperbolic type PDE

$$
\rho(x) y_{t t}=P(t) y_{x x}, \quad(x, t) \in Q_{T}=(0, l) \times(0, T),
$$

subject to the boundary conditions (BC)

$$
y_{x}(0, t)=\frac{f(t)}{P(t)}, y(l, t)=0,
$$


and the initial conditions (IC)

$$
y(x, 0)=y_{0}(x), y_{t}(x, 0)=y_{1}(x) .
$$

We assume

$$
P(t) \geq p_{0}>0, \quad \rho(x) \geq \rho_{0}>0
$$

with some positive constants $p_{0}$ and $\rho_{0}$. It is convenient to rename the function $f(t) / P(t)$ to $f(t)$ and, as usual in these matters, we call $f(t)$ to be the "control".

The paper is organized as follows. In Section 2 we discuss the statement of the problem and formulate the (known) results on the auxiliary Sturm-Liouville problem. We further describe the Liouville transformation, i.e. introduce "new time" and formulate the moment problem with respect to a system of functions in terms of that time. In Section 3 we prove the basis properties of that system of functions. We solve the moment problem and hence prove controllability of the system under consideration in Section 4. In Section 5 we sketch an extension of our results to controllability of an elastic ring. This extends the results obtained in [4] under more restrictive conditions on $P(t)$.

\section{Statement of IBVP. The problem of moments}

For any $T>0$, we consider IBVP (1.2)-(1.4). We assume condition (1.5) so that the functions $P(t)$ and $\rho(x)$ are strictly positive. Our goal is to find conditions on the system under consideration that guarantee the existence of the control $f(t)$ such that the string goes to rest in a given time $T$ and construct this control.

It is well-known (see [19]) that the initial boundary value problem (1.2) - (1.4) has a unique solution if the functions $P, \rho$, and $f$ are smooth enough.

To formulate the existence-uniqueness-regularity result for non-smooth coefficients, initial data and controls, we introduce the functional spaces $\mathcal{H}_{s}, s=0,1$ where $\mathcal{H}_{0}:=L^{2}(0, l)$ and the space $\mathcal{H}_{1}$ is defined to be a subspace of the Sobolev space $H^{1}(0, l), \mathcal{H}_{1}:=\left\{y \in H^{1}(0, l): y(l)=0\right\}$. Inner product and norm in $\mathcal{H}_{1}$ are as follows,

$$
\langle f, g\rangle_{\mathcal{H}_{1}}=\int_{0}^{l} f^{\prime}(s) \overline{g^{\prime}(s)} d s, \quad\|f\|_{\mathcal{H}_{1}}^{2}=\int_{0}^{l}\left|f^{\prime}(s)\right|^{2} \mathrm{~d} s .
$$

The following result holds. Its proof will be briefly discussed in Section 4 .

Theorem 1. Let $T>0$ and $f \in L^{2}(0, T), \rho \in C^{2}[0, l], P \in C^{2}[0, T], y_{0} \in \mathcal{H}_{1}, y_{1} \in \mathcal{H}_{0}$. Then there exists a unique (generalized) solution to IBVP (1.2)-(1.4) such that $y \in C\left([0, T] ; \mathcal{H}_{1}\right), y_{t} \in$ $C\left([0, T] ; \mathcal{H}_{0}\right)$.

We believe that the main results of this paper (Theorems 1 and 2) hold under less regularity assumptions on the coefficients: inclusions $\rho \in C^{1}[0, l]$ and $P \in C^{1}[0, T]$ would be sufficient; however, we do not discuss possible generalizations in this paper. 
Below $(\cdot, \cdot)$ denotes the inner product in $L^{2}(0, l)$ or $L^{2}(0, \tau)$ and $\|\cdot\|$ is the corresponding norm. Further, $(\cdot, \cdot)_{\rho}$ is the inner product in $L_{\rho}^{2}(0, l)$, and $\|\cdot\|_{\rho}$ is the corresponding norm.

We consider the following Sturm-Liouville problem (SLP),

$$
e^{\prime \prime}(x)+\lambda \rho(x) e(x)=0, \quad e^{\prime}(0)=e(l)=0 .
$$

Lemmas 1-5 below contain well-known and important for our purposes information about spectral properties of this problem. We suppose that $\rho \in C^{2}$ and $\rho>\rho_{0}$ with some positive constant $\rho_{0}$, although these results are valid under less regularity assumptions on $\rho$.

The following (and even stronger) result can be found in [19].

Lemma 1. The SLP (2.2) has a pure discrete positive spectrum $\left\{\lambda_{n}\right\}, n \geq 1$; the eigenfunctions $\left\{e_{n}(x)\right\}_{n \geq 1}$ form an orthogonal basis in $\mathcal{H}_{1}$ and $L_{\rho}^{2}(0, l)$,

$$
\left\langle e_{n}, e_{m}\right\rangle_{\mathcal{H}_{1}}=\left(e_{n}^{\prime}, e_{m}^{\prime}\right)=0,\left(e_{n}, e_{m}\right)_{\rho}=0 \text { if } n \neq m,
$$

and the identity holds,

$$
\left\|e_{n}\right\|_{\mathcal{H}_{1}}^{2}=\left\|e_{n}^{\prime}\right\|^{2}=\lambda_{n}\left\|e_{n}\right\|_{\rho}^{2} .
$$

It is convenient to assume below that the eigenfunctions are normalized in $L_{\rho}^{2}(0, l)$,

$$
\left\|e_{n}\right\|_{\rho}=1
$$

We mention also the following known result (see [5].)

Lemma 2. $u \in \mathcal{H}_{1}$ iff $u=\sum_{n} c_{n} e_{n}$, and $\sum_{n} \lambda_{n}\left|c_{n}\right|^{2}<\infty$. Also $\|u\|_{\mathcal{H}_{1}}^{2} \asymp \sum_{n} \lambda_{n}\left|c_{n}\right|^{2}$ (that may be understood as the equivalence of the corresponding norms.)

The following result may be proved with the help of the methods developed in [14]. Yet, we could not find the exact reference to the result and give a very brief proof.

Let

$$
\mathbf{L}:=\int_{0}^{l} \sqrt{\rho(s)} d s, \quad \sqrt{\lambda_{n}^{0}}:=\frac{\pi}{\mathbf{L}}\left(n-\frac{1}{2}\right), n \geq 1 .
$$

Lemma 3. (i) The system of functions

$$
e_{n}^{0}(x)=\sqrt{\frac{2}{\mathbf{L}}}(\rho(x))^{-1 / 4} \sin \left(\sqrt{\lambda_{n}^{0}} \int_{x}^{l} \sqrt{\rho(s)} d s\right), n \geq 1
$$

is orthonormal in $L_{\rho}^{2}(0, l)$.

(ii) The system of functions $\left\{e_{n}^{0}\right\}_{n \geq 1}$ forms a basis in $L_{\rho}^{2}(0, l)$. 
Proof. Only part (ii) requires brief comments. We introduce a new variable,

$$
\xi=\frac{\pi \int_{x}^{l} \sqrt{\rho(s)} d s}{\mathbf{L}}, \text { so that } d x=-\frac{\mathbf{L} d \xi}{\pi \sqrt{\rho(x)}},
$$

and note that the one-to-one transformation $x \rightarrow \xi$ maps the interval $(0, l)$ onto $(0, \pi)$. The functions

$$
\hat{e}_{n}^{0}(\xi):=\sqrt{\frac{2}{\pi}} \sin \left(n-\frac{1}{2}\right) \xi, \quad n \geq 1
$$

form an orthonormal basis in $L^{2}(0, \pi)$ (e.g. they are the eigenfunctions of SLP $y^{\prime \prime}+\lambda y=0, y(0)=$ $y^{\prime}(\pi)=0$.) We write the orthogonality relation for them using the change of variables (2.8),

$$
\begin{aligned}
& \left(\hat{e}_{n}^{0}, \hat{e}_{m}^{0}\right)_{L^{2}(0, \pi)}=\delta_{n}^{m} \\
& =\int_{0}^{l} \rho(x)\left[\sqrt{\frac{2}{\mathbf{L}}}(\rho(x))^{-1 / 4} \sin \left(\frac{\pi}{\mathbf{L}}\left(n-\frac{1}{2}\right) \int_{x}^{l} \sqrt{\rho(s)} d s\right) .\right. \\
& \left.\cdot \sqrt{\frac{2}{\mathbf{L}}}(\rho(x))^{-1 / 4} \sin \left(\frac{\pi}{\mathbf{L}}\left(m-\frac{1}{2}\right) \int_{x}^{l} \sqrt{\rho(s)} d s\right)\right] d x=\left(e_{n}^{0}, e_{m}^{0}\right)_{L_{\rho}^{2}(0, \pi)},
\end{aligned}
$$

where the functions $e_{n}^{0}(x)$ are given by (2.7). Hence, these functions form an orthonormal basis in $L_{\rho}^{2}(0, l)$.

Below we will need an asymptotic representation of the eigenfunctions $e_{n}(x)$ for large $n$. The following results are known (see [14].)

Lemma 4. The solutions of SLP (2.2) normalized by imposing condition (2.5) have the following asymptotic representation,

$$
e_{n}(x)=C_{n}(\rho(x))^{-1 / 4} \sin \left(\sqrt{\lambda_{n}} \int_{x}^{l} \sqrt{\rho(s)}\right) d s+O\left(\frac{1}{\sqrt{\lambda_{n}}}\right) .
$$

The constants $C_{n}$ are uniquely defined up to the sign, and the sequence $\left\{C_{n}\right\}$ is bounded.

The eigenvalues $\lambda_{n}$ satisfy the equation,

$$
r \sqrt{\lambda_{n}} \mathbf{L}=\tan \left(\sqrt{\lambda_{n}} \mathbf{L}\right)+O\left(\frac{1}{\sqrt{\lambda_{n}}}\right),
$$

where the constant $\mathbf{L}$ is as in (2.6) and the constant $r$ is introduced,

$$
r=-\frac{4(\rho(0))^{3 / 2}}{\rho^{\prime}(0)}
$$

The solution to Eq-n (2.10) satisfies

$$
\sqrt{\lambda_{n}}=\frac{\pi}{\mathbf{L}}\left(n-\frac{1}{2}\right)+O\left(\frac{1}{n}\right) \text { as } n \rightarrow \infty .
$$


Lemma 5. The following asymptotic relations hold,

$$
\begin{gathered}
\sqrt{\lambda_{n}}-\sqrt{\lambda_{n}^{0}}=O\left(\frac{1}{n}\right), \\
\left\|e_{n}-e_{n}^{0}\right\|_{L^{2}(0, l)}=O\left(\frac{1}{n}\right),\left|e_{n}(0)-(-1)^{n} C_{n}[\rho(0)]^{-1 / 4}\right|=O\left(\frac{1}{n}\right)
\end{gathered}
$$

with the constants $C_{n}$ from Lemma 4 .

We work in $L_{\rho}^{2}(0, l)$. We project PDE (1.2) on an arbitrary basis function $e_{n}(x)$, i.e. multiply that PDE on $e_{n}(x)$, integrate over $(0, l)$, and integrate by parts. We find,

$$
\frac{d^{2}}{d t^{2}}\left(y, e_{n}\right)_{\rho}=P(t)\left(\left.\left(y_{x} e_{n}-y e_{n}^{\prime}\right)\right|_{0} ^{l}+\left(y, e_{n}^{\prime \prime}\right)\right) .
$$

We use Eq-n (2.2) and BC for $y(x, t)$ and $e_{n}(x)$ to reduce Eq-n (2.15) to the form,

$$
\frac{d^{2}}{d t^{2}}\left(y, e_{n}\right)_{\rho}=-P(t) \lambda_{n}\left(y, e_{n}\right)_{\rho}-P(t) f(t) e_{n}(0) .
$$

We further denote

$$
Y_{n}(t)=\left(y(\cdot, t), e_{n}\right)_{\rho}
$$

to get the final form of Eq-n (2.16),

$$
\ddot{Y}_{n}(t)+\lambda_{n} P(t) Y_{n}(t)=-P(t) f(t) e_{n}(0) .
$$

In the similar way, the projected IC (1.4) have the form,

$$
Y_{n}(0)=\left(y_{0}, e_{n}\right)_{\rho}, \dot{Y}_{n}(0)=\left(y_{1}, e_{n}\right)_{\rho}, n \geq 1 .
$$

We now introduce the optical length of the string as the (positive) number $T_{0}$ such that

$$
\int_{0}^{T_{0}} \sqrt{P(t)} d t=\int_{0}^{l} \sqrt{\rho(x)} d x=\mathbf{L} .
$$

and the double optical length of the string as the (positive) number $T^{*}$ such that

$$
\int_{0}^{T^{*}} \sqrt{P(t)} d t=2 \int_{0}^{l} \sqrt{\rho(x)} d x .
$$

We notice that in general $T^{*} \neq 2 T_{0}$. 
It is shown in [2] that the characteristics of PDE (1.2) are given by the equations

$$
\int_{0}^{x} \sqrt{\rho\left(x^{\prime}\right)} d x^{\prime} \pm \int_{0}^{t} \sqrt{P\left(t^{\prime}\right)} d t^{\prime}=C .
$$

In particular, the line

$$
\int_{0}^{x} \sqrt{\rho\left(x^{\prime}\right)} d x^{\prime}-\int_{0}^{t} \sqrt{P\left(t^{\prime}\right)} d t^{\prime}=0
$$

is a characteristic and the points $(0,0)$ and $\left(l, T_{0}\right)$ belong to it. Hence, we may interpret $T_{0}$ as the time necessary for the signal to propagate from $x=0$ to $x=l$, and $T^{*}$ as the time necessary for the signal to propagate from $x=0$ to $x=l$ and back.

If we consider a string with the tension that is a function of coordinate $x$ only, we find the well-known formulas (see [28], [2], [5, Ch.V])

$$
T_{0}=\int_{0}^{l} \sqrt{\frac{\rho(x)}{P(x)}} d x, \quad T^{*}=2 T_{0} .
$$

The time $T^{*}$ plays an essential role in controllability theory. In our model, $T^{*}$ is the unique solution to Eq-n (2.21).

We consider the following controllability problem for IBVP (1.2)-(1.4).

Problem 1. Let a time $T>0$ be given. For any $\left(y_{0}, y_{1}\right) \in \mathcal{H}_{1} \times \mathcal{H}_{0}$, does there exist a control function $f \in L^{2}(0, T)$ such that the solution to the problem (1.2)-(1.4) satisfies the additional conditions at the time $t=T$,

$$
y(x, T)=y_{t}(x, T)=0, \quad x \in[0, l] ?
$$

The uniqueness of the solution (see Theorem 1) implies $y(x, t) \equiv 0$ for $t \geq T$ if (2.22) hold and $f(t)=0$ for $t>T$.

We will prove that the answer to this question is positive for $T \geq T^{*}$ and negative for $T<T^{*}$. This result looks similar to the well-known results about boundary controllability of a string with time-independent coefficients (see, e.g. [28], [5, Theorem V.2.9]), although the proof is more complicated.

Theorem 2. Consider IBVP (1.2)-(1.4). Let the conditions of Theorem 1 be satisfied. (A1) If $T \geq T^{*}$, then system (1.2)-(1.4) is exactly controllable, i.e. any initial data from $\mathcal{H}_{1} \times \mathcal{H}_{0}$ can be steered to zero.

(A2) If $T<T^{*}$, then the system is not even approximately controllable, i.e. the set of the initial data $\left(y_{0}, y_{1}\right)$ that may be driven to rest is not dense in $\mathcal{H}_{1} \times \mathcal{H}_{0}$. 
Remark 1. Another description of the states $\left(y_{0}, y_{1}\right)$ controllable in the time interval $\left[0, T^{*}\right]$ may be given in terms of a Hilbert space with the norm

$$
\left\|\left(y_{0}, y_{1}\right)\right\|^{2}:=\sum_{n} n^{2}\left|c_{n}^{0}\right|^{2}+\sum_{n}\left|c_{n}^{1}\right|^{2}
$$

and the corresponding inner product. From Lemma 2 it follows that this norm is equivalent to $\mathcal{H}_{1} \times \mathcal{H}_{0}$-norm. Such weighted $\ell^{2}-$ spaces are typical for control problems of distributed parameter systems (see [1], [4], [5].)

Remark 2. From the proof it will be clear that quite similar statements are valid for the description of the set of states reachable from zero initial data with the help of controls from $L^{2}(0, T)$. This fact is obvious for the wave equation with time independent coefficients but is not trivial in our case.

So far, we have reduced controllability problem to the study of the initial value problem (IVP) (2.18)-(2.19). It is obvious by linearity that the solution to this IVP is a linear combination of the projections of the initial data on the eigenfunctions (see (2.19)) and an integral containing $P(t) f(t)$ and two linearly independent solutions $\Phi_{n}(s), \Psi_{n}(s)$ of the homogeneous problem (2.18). Hence, controllability problem may be rewritten as the moment problem for the function $P(t) f(t)$ with respect to the system of functions $\left\{\Phi_{n}(s), \Psi_{n}(s)\right\}$. After the function $P(t) f(t)$ is found, the positivity of $P(t)$ allows to find the control $f(t)$ uniquely.

The solvability of the moment problem will be determined by the basis property of this system in $L^{2}(0,2 \mathbf{L})$ and will guarantee the solvability of controllability problem. The realization of this program is described in the rest of the paper.

We use Liouville transformation that is the common tool in the asymptotic analysis of the second order ODE (see [14], Ch.IV, 4.1.) We introduce the new variable

$$
s=\int_{0}^{t} \omega(\tau) d \tau \text { where } \omega(t)=\sqrt{P(t)} .
$$

We note that the transformation $t \rightarrow s$ of the interval $[0, T]$ onto the interval $[0, S], S:=s(T)$, is one-to-one and hence, (2.23) defines a function

$$
t=t(s), t \in C^{3}[0, S] .
$$

We also introduce the new unknown function $z_{n}(s)$ by the formula,

$$
Y_{n}(t)=\omega^{-1 / 2}(t) z_{n}(s) .
$$

Applying the transformation (2.23), (2.25) to ODE (2.18) yields the new ODE,

$$
z_{n}^{\prime \prime}+\left(Q(s)+\lambda_{n}\right) z_{n}=e_{n}(0) F(s),
$$

where the following functions are introduced,

$$
Q(s)=\frac{3 \dot{\omega}(t)^{2}}{4 \omega^{4}(t)}-\frac{\ddot{\omega}(t)}{2 \omega^{3}(t)} \text { and } F(s)=-\frac{P(t) f(t)}{\omega(t)^{3 / 2}} \text { with } t=t(s) .
$$


We note that the properties of $\omega(t)$ and $f(t)$ imply the following inclusions,

$$
Q \in C[0, S] \text { and } F \in L^{2}(0, S) .
$$

We also note that the function $Q(s)$ (which is called Liouville transformation of $P(t)$ ) is "small" if $P(t)$ (or $\omega(t)=\sqrt{P(t)}$ ) varies "slowly"; the last fact was very important for the proof in [1], [4] but is not used at all here.

Applying the transformation (2.23), (2.25) to IC (1.4) yields the following initial conditions for $z_{n}(s)$

$$
\begin{gathered}
z_{n}(0)=z_{n}^{0}, \quad z_{n}^{\prime}(0)=z_{n}^{1} \text { where } \\
z_{n}^{0}=\omega^{1 / 2}(0)\left(y_{0}, e_{n}\right)_{\rho}, \quad z_{n}^{1}=\omega^{-1 / 2}(0)\left(y_{1}, e_{n}\right)_{\rho}+\frac{1}{2} \omega^{-3 / 2}(0) \dot{\omega}(0)\left(y_{0}, e_{n}\right)_{\rho} .
\end{gathered}
$$

The solution to the initial value problem (2.26), (2.29) may be given in terms of two independent solutions of the corresponding homogeneous ODE,

$$
\tilde{z}_{n}^{\prime \prime}+\left(Q(s)+\lambda_{n}\right) \tilde{z}_{n}=0 .
$$

We introduce two solutions $\Phi_{n}(t)$ and $\Psi_{n}(t)$ of Eq-n (2.30) satisfying different initial conditions,

$$
\begin{gathered}
\Phi_{n}^{\prime \prime}+\left(Q(s)+\lambda_{n}\right) \Phi_{n}=0, \quad \Phi_{n}(0)=0, \quad \Phi_{n}^{\prime}(0)=\sqrt{\lambda_{n}} \\
\text { and } \Psi_{n}^{\prime \prime}+\left(Q(s)+\lambda_{n}\right) \Psi_{n}=0, \quad \Psi_{n}(0)=1, \quad \Psi_{n}^{\prime}(0)=0 .
\end{gathered}
$$

Obviously these two solutions are linearly independent (their Wronskian is $-\sqrt{\lambda_{n}}$ ). Then the (unique) solution to the problem (2.26), (2.29) has the form,

$$
\begin{gathered}
z_{n}(s)=\frac{1}{\sqrt{\lambda_{n}}} z_{n}^{1} \Phi_{n}(s)+z_{n}^{0} \Psi_{n}(s) \\
+\frac{e_{n}(0)}{\sqrt{\lambda_{n}}} \int_{0}^{s}\left(\Phi_{n}(s) \Psi_{n}(\tau)-\Phi_{n}(\tau) \Psi_{n}(s)\right) F(\tau) d \tau .
\end{gathered}
$$

We also find

$$
\begin{gathered}
z_{n}^{\prime}(s)=\frac{1}{\sqrt{\lambda_{n}}} z_{n}^{1} \Phi_{n}^{\prime}(s)+z_{n}^{0} \Psi_{n}^{\prime}(s) \\
+\frac{e_{n}(0)}{\sqrt{\lambda_{n}}} \int_{0}^{s}\left(\Phi_{n}^{\prime}(s) \Psi_{n}(\tau)-\Phi_{n}(\tau) \Psi_{n}^{\prime}(s)\right) F(\tau) d \tau .
\end{gathered}
$$

We are looking for a boundary control $f(t)$ (or $F(s)$ ) that drives the solution $y(x, t)$ to rest in the given time $T$, i.e. according to Eq-n (2.17),

$$
Y_{n}(T)=\dot{Y}_{n}(T)=0 \text { for all } n \geq 1
$$

According to Eq-n (2.23), the last requirement, in terms of the transformation (2.25), leads to the conditions

$$
z_{n}(S)=z_{n}^{\prime}(S)=0, \quad S=s(T)
$$


The number $S$ for which controllability holds will be specified in Section 4; this $S$ is equal to $2 \mathbf{L}=s\left(T^{*}\right)$.

Substituting $s=S$ into representation (2.33) and (2.34) and using conditions (2.36) leads to the following equations for the control $F(s)$ that guarantee the exact controllability of the string,

$$
\begin{gathered}
\int_{0}^{S}\left(\Phi_{n}(S) \Psi_{n}(\tau)-\Phi_{n}(\tau) \Psi_{n}(S)\right) F(\tau) d \tau \\
=-\frac{1}{e_{n}(0)}\left(z_{n}^{1} \Phi_{n}(S)+\sqrt{\lambda_{n}} z_{n}^{0} \Psi_{n}(S)\right), \\
\int_{0}^{S}\left(\Phi_{n}^{\prime}(S) \Psi_{n}(\tau)-\Phi_{n}(\tau) \Psi_{n}^{\prime}(S)\right) F(\tau) d \tau \\
=-\frac{1}{e_{n}(0)}\left(z_{n}^{1} \Phi_{n}^{\prime}(S)+\sqrt{\lambda_{n}} z_{n}^{0} \Psi_{n}^{\prime}(S)\right), \quad n \geq 1 .
\end{gathered}
$$

We rewrite the last two equations in the form,

$$
\mathcal{A}_{n} \mathcal{F}_{n}=\mathcal{G}_{n}, \quad n \geq 1
$$

where the matrices and vectors are introduced,

$$
\begin{gathered}
\mathcal{A}_{n}=\left[\begin{array}{ll}
\Phi_{n}(S) & -\Psi_{n}(S) \\
\Phi_{n}^{\prime}(S) & -\Psi_{n}^{\prime}(S)
\end{array}\right], \mathcal{F}_{n}=\int_{0}^{S}\left[\begin{array}{r}
\Psi_{n}(\tau) \\
\Phi_{n}(\tau)
\end{array}\right] F(\tau) d \tau \\
\text { and } \mathcal{G}_{n}=-\frac{1}{e_{n}(0)}\left[\begin{array}{l}
z_{n}^{1} \Phi_{n}(S)+\sqrt{\lambda_{n}} z_{n}^{0} \Psi_{n}(S) \\
z_{n}^{1} \Phi_{n}^{\prime}(S)+\sqrt{\lambda_{n}} z_{n}^{0} \Psi_{n}^{\prime}(S)
\end{array}\right]
\end{gathered}
$$

The linear algebraic system (2.39) has a unique solution because of the linear independence of $\Phi_{n}$ and $\Psi_{n}$. Solving it yields the final form of the conditions on the control $F$ that guarantee the exact controllability,

$$
\int_{0}^{S} \Psi_{n}(\tau) F(\tau) d \tau=-\frac{z_{n}^{1}}{e_{n}(0)}, \quad \int_{0}^{S} \Phi_{n}(\tau) F(\tau) d \tau=\frac{\sqrt{\lambda_{n}}}{e_{n}(0)} z_{n}^{0}, \quad n \geq 1 .
$$

We note that $e_{n}(0) \neq 0$ for every $n$, as it is obvious from the problem (2.2). Furthermore, due to the second of the estimates (2.14), the sequences $\left\{z_{n}^{0} / e_{n}(0)\right\}$ and $\left\{z_{n}^{1} / e_{n}(0)\right\}$ belong to $\ell^{2}$ and have the same asymptotic behavior as the sequences $\left\{z_{n}^{0}\right\}$ and $\left\{z_{n}^{1}\right\}$. So, in order to study our problem, we can simply rename

$$
z_{n}^{0}:=z_{n}^{0} / e_{n}(0), \quad z_{n}^{1}:=z_{n}^{1} / e_{n}(0) .
$$

The exact controllability of our model is now equivalent to the solvability of the following moment problem,

$$
\int_{0}^{S} \Psi_{n}(\tau) F(\tau) d \tau=-z_{n}^{1}, \quad \int_{0}^{S} \Phi_{n}(\tau) F(\tau) d \tau=\sqrt{\lambda_{n}} z_{n}^{0}, \quad n \geq 1 .
$$


This is the problem we are going to study.

It appears to be more convenient to go over to another pair of equations equivalent to (2.42). We introduce the functions $\Gamma_{n}^{ \pm}$as the (unique) solutions to the Cauchy problem

$$
\left(\Gamma_{n}^{ \pm}\right)^{\prime \prime}+\left(Q(s)+\lambda_{n}\right) \Gamma_{n}^{ \pm}=0, \Gamma_{n}^{ \pm}(0)=1, \quad\left(\Gamma_{n}^{ \pm}\right)^{\prime}(0)= \pm i \sqrt{\lambda_{n}} .
$$

Obviously the relation holds,

$$
\Gamma_{n}^{ \pm}(s)=\Psi_{n}(s) \pm i \Phi_{n}(s), \quad n \geq 1 .
$$

The conditions of controllability (2.42) may now be rewritten in the form of a moment problem,

$$
\int_{0}^{S} F(\tau) \Gamma_{n}^{ \pm}(\tau) d \tau=-z_{n}^{1} \pm i \sqrt{\lambda_{n}} z_{n}^{0}, \quad n \geq 1
$$

The moment problem (2.45) represents the main object of our consideration.

Remark 3. If the function $P$ (and therefore $\omega$ ) is a constant, and hence, $Q(s) \equiv 0$ (see (2.27)), we easily find

$$
\Phi_{n}(s)=\sin \sqrt{\lambda_{n}} s, \quad \Psi_{n}(s)=\cos \sqrt{\lambda_{n}} s, \quad \Gamma_{n}^{ \pm}(s)=e^{ \pm i \sqrt{\lambda_{n}}} .
$$

Then the moment problem (2.45) has the form of the classical moment problem,

$$
\int_{0}^{S} e^{ \pm i \sqrt{\lambda_{n}} \tau} F(\tau) d \tau=-z_{n}^{1} \pm i \sqrt{\lambda_{n}} z_{n}^{0} \quad n \geq 1 .
$$

The result on controllability (Theorem 2) is known in this case (see, e.g. [5], Ch.V.) Yet, in our problem, the function $P(t)$ is not a constant, and the moment problem appears to be much more complicated.

\section{Basis properties of the system $\left\{\Gamma_{n}^{ \pm}(s)\right\}$}

In [1], [4] the basis property of the system $\left\{\Gamma_{n}^{ \pm}(s)\right\}$ is proved with the help of the Krein theorem about a basis that is quadratically close to an orthonormal one (see [15].) It is important for the proofs in both papers that the tension $P(t)$ changes "slowly" with time (to be precise, its Liouville transformation is supposed to be small.) The proof below is based on a completely different idea and does not contain any requirement on the Liouville transformation of the tension.

We consider the basis properties of the family of the functions $\left\{\Gamma_{n}^{ \pm}\right\}$that are (unique) solutions to the Cauchy problem (2.43). The functions $\Gamma_{n}^{ \pm}$satisfy the integral equation (IE),

$$
\Gamma_{n}^{ \pm}(\tau)=e^{ \pm i \sqrt{\lambda_{n}} \tau}-\frac{1}{\sqrt{\lambda_{n}}} \int_{0}^{\tau} \sin \sqrt{\lambda_{n}}(\tau-s) Q(s) \Gamma_{n}^{ \pm}(s) d s .
$$

We remind that the transformation $t \rightarrow s(t)$ defined in (2.23) maps the interval $\left[0, T^{*}\right]$ onto the interval $[0,2 \mathbf{L}]$, where $\mathbf{L}$ and $T^{*}$ are introduced by (2.6) and (2.21) correspondingly.

Our goal in this section is to prove the following basis property result. 
Lemma 6. The family $\Gamma:=\left\{\Gamma_{n}^{ \pm}(\tau)\right\}$ forms a Riesz basis in $L^{2}(0,2 \mathbf{L})$.

The proof is given below and is based on the following well-known theorem by N. Bari (see, e.g. [15].)

Theorem (Bari). If a sequence of vectors in a Hilbert space $\mathcal{H}$ is $\omega$-linearly independent and quadratically close to a Riesz basis of $\mathcal{H}$, then that sequence is a Riesz basis of $\mathcal{H}$ itself.

We remind here that a sequence of almost normalized vectors $\left\{g_{j}\right\}_{j \geq 1}$ is called $\omega$-linearly independent, if the equality

$$
\sum_{j \geq 1} c_{j} g_{j}=0
$$

is impossible for any nontrivial sequence $\left\{c_{j}\right\} \in \ell^{2}$.

Hence, the basis property is proved if we prove two results.

(a) The sequence $\left\{\Gamma_{n}^{ \pm}(s)\right\}$ is quadratically close to the sequence $\left\{e^{ \pm i \sqrt{\lambda_{n}^{0}} s}\right\}$ in $L^{2}(0,2 \mathbf{L})$ and

(b) The sequence $\left\{\Gamma_{n}^{ \pm}(s)\right\}$ is $\omega$-linearly independent in $L^{2}(0,2 \mathbf{L})$.

Lemma 7. (A1) The sequence $\left\{\Gamma_{n}^{ \pm}(s)\right\}$ is quadratically close in $L^{2}(0,2 \mathbf{L})$ both to the sequence $\left\{e^{ \pm i \sqrt{\lambda_{n}^{0}} s}\right\}$ and to the sequence $\left\{e^{ \pm i \sqrt{\lambda_{n}} s}\right\}$.

(A2) There exists a (positive) integer number $N_{*}$ such that the sequence $\left\{\Gamma_{n}^{ \pm}(s)\right\}_{n \geq N_{*}}$ is an $\mathcal{L}$-basis in $L^{2}(0,2 \mathbf{L})$.

Proof. (A1) It is easily seen from Eq-n (3.1) and the Gronwall inequality [31] that the sequence $\left\{\Gamma_{n}^{ \pm}(s)\right\}$ is bounded on $[0,2 \mathbf{L}]$. Then according to Eq-n (3.1), this sequence is quadratically close to the sequence $\left\{e^{ \pm i \sqrt{\lambda_{n}} s}\right\}$ in $L^{2}(0,2 \mathbf{L})$. Further, according to (2.13), the sequence $\left\{e^{ \pm i \sqrt{\lambda_{n}} s}\right\}$ is quadratically close to the sequence $\left\{e^{ \pm i \sqrt{\lambda_{n}^{0}} s}\right\}$ in $L^{2}(0,2 \mathbf{L})$. The triangle inequality completes the proof of (A1).

(A2) Statement (A1) implies that, for sufficiently large $N_{*}$, there exists a linear bounded and boundedly invertible operator in $L^{2}(0,2 \mathbf{L})$ which maps the sequence

$$
\left\{e^{ \pm i \sqrt{\lambda_{n}^{0}} s}\right\}_{n \geq 1} \text { to }\left\{e^{ \pm i \sqrt{\lambda_{n}^{0}} s}\right\}_{n<N_{*}} \bigcup\left\{\Gamma_{n}^{ \pm}(s)\right\}_{n \geq N_{*}}
$$

Therefore, the latter sequence forms a Riesz basis in $L^{2}(0,2 \mathbf{L})$, and so $\left\{\Gamma_{n}^{ \pm}(s)\right\}_{n \geq N_{*}}$ is an $\mathcal{L}$-basis in this space.

We will need also the following auxiliary result.

Lemma 8. The family $\left\{\Gamma_{n}^{ \pm}(s)\right\}$ is linearly independent in $L^{2}(0,2 \mathbf{L})$.

Proof. Let

$$
\sum_{ \pm} \sum_{n=1}^{M} c_{n}^{ \pm} \Gamma_{n}^{ \pm}(s)=0
$$

for some finite $M \geq 1$. Differentiating Eq-n (3.2) twice and using ODE (2.30) yields,

$$
\sum_{ \pm} \sum_{j}^{M} c_{n}^{ \pm} \lambda_{n} \Gamma_{n}^{ \pm}(s)=0 .
$$


We may repeat the same operation several times to get,

$$
\sum_{ \pm} \sum_{n=1}^{M} c_{n}^{ \pm} \lambda_{n}^{k} \Gamma_{n}^{ \pm}(s)=0, \quad k=0,1, \ldots, M-1
$$

The notation

$$
\eta_{n}=\sum_{ \pm} c_{n}^{ \pm} \Gamma_{n}^{ \pm}(s), \quad 1 \leq n \leq M
$$

allows to view the series of conditions (3.4) as a linear algebraic $M \times M$-system with Vandermonde matrix $\left\{\lambda_{n}^{k}\right\}, 0 \leq k \leq M-1,1 \leq n \leq M$. Hence, all $\eta_{n}=0$. Linear independence of the two functions $\Gamma_{n}^{+}$and $\Gamma_{n}^{-}$(for every fixed index $n$ ) implies $c_{n}^{+}=0$ and $c_{n}^{-}=0$.

In the course of the proof below, it is convenient to avoid double sums. So, in this proof we set:

$$
\Gamma_{n}:=\Gamma_{n}^{+}, \quad \Gamma_{-n}:=\Gamma_{n}^{-}, \quad \sqrt{\lambda_{-n}}:=-\sqrt{\lambda_{n}}, \quad \lambda_{-n}:=\lambda_{n}, \quad n \in \mathbb{N},
$$

and in what follows, we suppose that $n$ runs over $\mathbb{Z}^{\prime}:= \pm 1, \pm 2, \ldots \quad$ Also, we denote $\ell^{2}\left(\mathbb{Z}^{\prime}\right)$ simply as $\ell^{2}$.

As one could expect, the following result requires much work. Its proof extends to the end of this section.

Lemma 9. The sequence $\left\{\Gamma_{n}(s)\right\}$ is $\omega$-linearly independent in $L^{2}(0,2 \mathbf{L})$.

Proof. First, we note that the sequence $\left\{\Gamma_{n}(s)\right\}$ is bounded on $[0,2 \mathbf{L}]$ (see the proof of Lemma 7.)

We proceed by contradiction, i.e. assume that there exists a nontrivial sequence

$$
\left\{\alpha_{n}\right\} \in \ell^{2}
$$

such that

$$
\sum_{n \in \mathbb{Z}^{\prime}} \alpha_{n} \Gamma_{n}(s)=0
$$

The series converges in $L^{2}(0,2 \mathbf{L})$. We further introduce the auxiliary function $g(\tau)$ as follows,

$$
g(\tau)=\sum_{n \in \mathbb{Z}^{\prime}} \frac{\alpha_{n}}{\sqrt{\lambda_{n}}} \int_{0}^{\tau} \sin \sqrt{\lambda_{n}}(\tau-s) Q(s) \Gamma_{n}(s) d s, \tau \in[0,2 \mathbf{L}] .
$$

Eq-s (3.1) and (3.7) now imply,

$$
g(\tau)=\sum_{n \in \mathbb{Z}^{\prime}} \alpha_{n} e^{i \sqrt{\lambda_{n}} \tau}
$$

Both representations (3.8) and (3.9) will be used below.

The embedding (3.6) shows that the series (3.9) for $g(\tau)$ converges in $L^{2}(0,2 \mathbf{L})$ but the series (3.8) converges uniformly so that $g(\tau)$ is a continuous function.

Now we proceed in three steps. 
- We prove that $g \in H^{1}(0,2 \mathbf{L})$.

We compute the derivative of the series (3.8) termwise and then prove that the resulting series converges in $L^{2}(0,2 \mathbf{L})$. Termwise differentiation of (3.8) yields,

$$
g^{\prime}(\tau) \sim \sum_{n \in \mathbb{Z}^{\prime}} \alpha_{n} \int_{0}^{\tau} \cos \sqrt{\lambda_{n}}(\tau-s) Q(s) \Gamma_{n}(s) d s .
$$

Here and below the symbol $\sim$ means the series in the RHS appears as a result of the formal differentiation.

We now show that the series (3.10) converges in $L^{2}(0,2 \mathbf{L})$. First, we rewrite the series as follows,

$$
\begin{gathered}
\sum_{n \in \mathbb{Z}^{\prime}} \alpha_{n} \int_{0}^{\tau} \cos \sqrt{\lambda_{n}}(\tau-s) Q(s) \Gamma_{n}(s) d s \\
=\sum_{n \in \mathbb{Z}^{\prime}} \alpha_{n} \int_{0}^{\tau} \cos \sqrt{\lambda_{n}}(\tau-s) Q(s)\left[\Gamma_{n}(s)-e^{i \sqrt{\lambda_{n}} s}\right] d s \\
+\frac{1}{2}\left[\int_{0}^{\tau} Q(s) d s\right]\left[\sum_{n \in \mathbb{Z}^{\prime}} \alpha_{n} e^{i \sqrt{\lambda_{n}} \tau}\right] \\
+\frac{1}{2} \sum_{n \in \mathbb{Z}^{\prime}} \alpha_{n} e^{-i \sqrt{\lambda_{n}} \tau} \int_{0}^{\tau} Q(s) e^{2 i \sqrt{\lambda_{n}} s} d s .
\end{gathered}
$$

The series in (3.11) converges uniformly due to Lemma 7. The asymptotic formula (2.12) implies (see, e.g. [5, Sec. II.4]) that the family $\left\{e^{i \sqrt{\lambda_{n}} t}\right\}$ forms a Riesz basis in $L^{2}(0,2 \mathbf{L})$. Therefore, the series in (3.12) converges in $L^{2}(0,2 \mathbf{L})$. By the same reason, $\left|\int_{0}^{\tau} Q(s) e^{2 i \sqrt{\lambda_{n}} s} d s\right| \leq q_{n}$ with a square integrable sequence $\left\{q_{n}\right\}$. We conclude that the series (3.13) converges uniformly, and the series (3.10) converges in $L^{2}(0,2 \mathbf{L})$.

Hence, $g(\tau)$ and $g^{\prime}(\tau)$ are square integrable. We arrive at the embedding $g \in H^{1}(0,2 \mathbf{L})$.

Since the family $\left\{e^{i \sqrt{\lambda_{n}} t}\right\}$ forms a Riesz basis in $L^{2}(0,2 \mathbf{L})$, the family $\left\{\frac{e^{i \sqrt{\lambda_{n}}} t}{\sqrt{\lambda_{n}}}\right\}$ is an $\mathcal{L}$-basis in the space $H^{1}(0,2 \mathbf{L})$ (see [29], [5]). Then Eq-n (3.9) may be rewritten as follows,

$$
g(\tau)=\sum_{n \in \mathbb{Z}^{\prime}} \alpha_{n} \sqrt{\lambda_{n}} \frac{e^{i \sqrt{\lambda_{n}} \tau}}{\sqrt{\lambda_{n}}},
$$

and the inclusion $g \in H^{1}(0,2 \mathbf{L})$ implies that $\alpha_{n}=\sigma_{n} / \sqrt{\lambda_{n}}$ with $\left\{\sigma_{n}\right\} \in \ell^{2}$.

- We now prove that $g \in H^{2}(0,2 \mathbf{L})$.

Using the result in the previous step, we may rewrite the function $g$ as follows,

$$
g(\tau)=\sum_{n \in \mathbb{Z}^{\prime}} \frac{\sigma_{n}}{\sqrt{\lambda_{n}}} e^{i \sqrt{\lambda_{n}} \tau}=\sum_{n \in \mathbb{Z}^{\prime}} \frac{\sigma_{n}}{\lambda_{n}} \int_{0}^{\tau} \sin \sqrt{\lambda_{n}}(\tau-s) Q(s) \Gamma_{n}(s) d s .
$$


We may differentiate both sides due to the inclusion $g \in H^{1}(0,2 \mathbf{L})$ to get,

$$
g^{\prime}(\tau)=i \sum_{n \in \mathbb{Z}^{\prime}} \sigma_{n} e^{i \sqrt{\lambda_{n}} \tau}=\sum_{n \in \mathbb{Z}^{\prime}} \frac{\sigma_{n}}{\sqrt{\lambda_{n}}} \int_{0}^{\tau} \cos \sqrt{\lambda_{n}}(\tau-s) Q(s) \Gamma_{n}(s) d s,
$$

where both series converge in $L^{2}(0,2 \mathbf{L})$ due to the embedding $\left\{\sigma_{n}\right\} \in \ell^{2}$ and the aforementioned properties of $\lambda_{n}$ and $\Gamma_{n}$. We differentiate the series on the right-hand-side of (3.15) once more to get,

$$
\begin{gathered}
g^{\prime \prime}(\tau) \sim Q(\tau) \sum_{n \in \mathbb{Z}^{\prime}} \frac{\sigma_{n}}{\sqrt{\lambda_{n}}} \Gamma_{n}(\tau) \\
-\sum_{n \in \mathbb{Z}^{\prime}} \sigma_{n} \int_{0}^{\tau} \sin \sqrt{\lambda_{n}}(\tau-s) Q(s) \Gamma_{n}(s) d s=: \Sigma_{1}+\Sigma_{2} .
\end{gathered}
$$

Here the series $\Sigma_{1}=0$ by assumption of Lemma 9. A procedure similar to one we used for the series (3.10) (which was based on the properties of $\sigma_{n}, \lambda_{n}$, and $\Gamma_{n}$ ) proves that the series $\Sigma_{2}$ converges in $L^{2}(0,2 \mathbf{L})$.

This completes the proof that $g \in H^{2}(0,2 \mathbf{L})$.

- We finally prove Lemma 9.

We proceed as in the previous step using the fact that the set $\left\{\frac{e^{i \sqrt{\lambda_{n}}} t}{\lambda_{n}}\right\}$ is an $\mathcal{L}$-basis in the space $H^{2}(0,2 \mathbf{L})$ (see [29], [5]), to find that the inclusion $g \in H^{2}(0,2 \mathbf{L})$ implies that

$$
\alpha_{n}=\frac{\mu_{n}}{\lambda_{n}} \text { with }\left\{\mu_{n}\right\} \in \ell^{2} .
$$

Eq-n (3.7) now implies,

$$
\sum_{n \in \mathbb{Z}^{\prime}} \frac{\mu_{n}}{\lambda_{n}} \Gamma_{n}(\tau)=0
$$

We further differentiate Eq-n (3.7) (or (3.17)) termwise. We find from Eq-n (3.1)

$$
\Gamma_{n}^{\prime}(\tau)=i \sqrt{\lambda_{n}} e^{i \sqrt{\lambda_{n}} \tau}-\int_{0}^{\tau} \cos \sqrt{\lambda_{n}}(\tau-s) Q(s) \Gamma_{n}(s) d s
$$

Formal differentiation of the series (3.17) gives, with the help of (3.18),

$$
\sum_{n \in \mathbb{Z}^{\prime}} \frac{\mu_{n}}{\lambda_{n}}\left(i \sqrt{\lambda_{n}} e^{i \sqrt{\lambda_{n}} \tau}-\int_{0}^{\tau} \cos \sqrt{\lambda_{n}}(\tau-s) Q(s) \Gamma_{n}(s) d s\right)=0,
$$

where the series converges uniformly.

We further differentiate Eq-n (3.17) termwise twice; the new series converges in $L^{2}(0,2 \mathbf{L})$. We use differential Eq-n (2.43) to find,

$$
\begin{aligned}
& 0=\sum_{n \in \mathbb{Z}^{\prime}} \frac{\mu_{n}}{\lambda_{n}} \Gamma_{n}^{\prime \prime}(\tau)=\sum_{n \in \mathbb{Z}^{\prime}} \frac{\mu_{n}}{\lambda_{n}}\left[-\lambda_{n} \Gamma_{n}(t)-Q(\tau) \Gamma_{n}(\tau)\right] \\
& =-\sum_{n \in \mathbb{Z}^{\prime}} \mu_{n} \Gamma_{n}(\tau)-Q(\tau) \sum_{n \in \mathbb{Z}^{\prime}} \frac{\mu_{n}}{\lambda_{n}} \Gamma_{n}(\tau) .
\end{aligned}
$$


Here we split the series into the sum of two series since the last series converges uniformly and the first series converges in $L^{2}(0,2 \mathbf{L})$. In fact, the second series converges to zero due to Eq-n (3.7). Hence, we arrive at the identity,

$$
\sum_{n \in \mathbb{Z}^{\prime}} \mu_{n} \Gamma_{n}(\tau)=0
$$

where convergence is in $L^{2}(0,2 \mathbf{L})$-sense. We now use Eq-n (3.16) to get,

$$
\sum_{n \in \mathbb{Z}^{\prime}} \alpha_{n} \lambda_{n} \Gamma_{n}(\tau)=0
$$

This equality coupled with (3.7) results in the identity,

$$
\sum_{|n| \geq 2} \mu_{n}^{(1)} \Gamma_{n}(\tau)=0 \text { where }\left\{\mu_{n}^{(1)}\right\}=\left\{\left(1-\frac{\lambda_{1}}{\lambda_{n}}\right) \mu_{n}\right\} \in \ell^{2} .
$$

Here convergence is in $L^{2}(0,2 \mathbf{L})$-sense.

Furthermore, $\mu_{n}^{(1)}$ is zero iff $\mu_{n}$ is zero, i.e. iff $\alpha_{n}$ is zero.

Hence, we have a condition similar to (3.7), with two terms corresponding to $n= \pm 1$ removed.

We can iterate the process and, after a finite number of steps, we end up with a condition

$$
\sum_{|n| \geq N_{*}} \tilde{\mu}_{n} \Gamma_{n}(\tau)=0
$$

This shows that $\tilde{\mu}_{n}=0$ for every $n,|n| \geq N_{*}$, since $\left\{\Gamma_{n}(s)\right\}_{|n| \geq N_{*}}$ is an $\mathcal{L}$-basis. Hence, $\alpha_{n}=0$ for every $n,|n| \geq N_{*}$. Finally, the linear independence of $\left\{\Gamma_{n}(s)\right\}$ (Lemma 8) shows that $\alpha_{n}=0$ for every $n,|n|<N_{*}$, and this completes the proof of $\omega$-linear independence.

\section{Moment problem. Controllability}

We consider the moment problem (2.45) with $S=2 \mathbf{L}$ that corresponds to $T=T^{*}$. We introduce the sequence

$$
\zeta_{n}=-z_{|n|}^{1} \mp i \sqrt{\lambda_{n}} z_{|n|}^{0}, \quad n \in \mathbb{Z}^{\prime} .
$$

Then Eq-ns (2.45) may be rewritten as follows,

$$
\left(F, \Gamma_{n}\right)=\zeta_{n}, \quad n \in \mathbb{Z}^{\prime}
$$

Here $(\cdot, \cdot)$ denotes the inner product in $L^{2}(0,2 \mathbf{L})$.

We introduce the basis $\left\{\gamma_{n}\right\}$ that is biorthogonal to the basis $\left\{\Gamma_{n}\right\}$, i.e.

$$
\left(\Gamma_{n}, \gamma_{m}\right)=\delta_{n}^{m} \text { for any } n, m \in \mathbb{Z}^{\prime} .
$$


The conditions (4.2) mean that $\left\{\zeta_{n}\right\}$ are the Fourier coefficients of $F$ with respect to the basis $\left\{\Gamma_{n}\right\}$ and hence, the function $F$ may be reconstructed as follows,

$$
F(s)=\sum_{n}\left(F, \Gamma_{n}\right) \gamma_{n}(s)=\sum_{n} \zeta_{n} \gamma_{n}(s)
$$

Proof of Theorem 1.

Since we have proved that the family $\left\{\Gamma_{n}\right\}$ forms a Riesz basis in $L^{2}(0,2 \mathbf{L})$, the proof of Theorem 1 is a minor variation of the corresponding proofs at [1] and [5, Secs. III.2,V.2].

Proof of Theorem 2.

(A1) Exact controllability of the system (1.2)-(1.4) in the time interval $[0, T]$ is equivalent to solvability of the moment problem (2.45) with $S=S(T)$. Statement A1 is the direct consequence of the fact that the family $\left\{\Gamma_{n}\right\}$ forms a Riesz basis in $L^{2}(0,2 \mathbf{L})$.

(A2) If $T<T^{*}$, then $S(T)<2 \mathbf{L}$, and there exists a proper subfamily of $\left\{e^{i \sqrt{\lambda_{n}} s}\right\}$ which forms a Riesz basis in $L^{2}(0, S)$ (see [5], Theorem II.4.16.) Moreover, this subfamily, say $\widetilde{\Gamma}^{N}$, may be chosen from the family $\left\{e^{i \sqrt{\lambda_{n}} s}\right\}_{|n| \geq N}$ for any prescribed $N$. The corresponding subfamily $\Gamma^{N}$ of $\Gamma$ also forms a Riesz basis in $L^{2}(0, S)$. Indeed, the operator that transforms $\widetilde{\Gamma}^{N}$ to $\Gamma^{N}$ is boundedly invertible if $N$ is big enough. Then according to [5, Theorem I.2.1(e)], the system (1.2)-(1.4) is not approximately controllable.

\section{Controllability of an elastic ring}

The exact controllability of an elastic ring under the stretching tension that varies in time is studied in [4]. It is shown that applying two independent exterior controls is necessary for controllability. A technical assumption that the tension varies slowly is used in [4]. Our approach here can be used to remove such assumption.

To simplify the presentation, we consider the case of homogeneous ring as in [4]. We omit details and assumptions if they are the same as for elastic string (Sections 2, 3, and 4) and only show a sketch of the proof.

\subsection{The model. Main assumptions}

An elastic ring represents an important element of many engineering constructions. We mention here only few technical applications of the model. (a) Vibrations of an elastic ring are considered in [22] in connection with elastic wheels for train wagons (which could replace the usual steel wheels.) It is easy to show (see [4]) that the equation for the circumferential displacement has the form of the wave equation, i.e. formally coincides with the equation describing transverse vibrations of a string subject to periodic boundary conditions. (b) Vibrations of elastic shells are of permanent interest to engineers (see, e.g. [30], [33].) An elastic ring may be viewed as a short cylindrical shell. If oscillations are independent on the longitudinal coordinates, the equation for 
the longitudinal displacement also coincides with the equation describing a string subject to periodic boundary conditions. (c) The problem of oscillations of an infinite string under the periodic load may be reduced to the problem for one period.

For any $T>0$, we consider IBVP

$$
\rho y_{t t}=P(t) y_{x x}+\rho \sum_{j=1}^{2} g^{j}(x) f_{j}(t), \quad(x, t) \in(0, l) \times(0, T),
$$

subject to periodic BC

$$
y(0, t)=y(l, t), \quad y_{x}(0, t)=y_{x}(l, t)
$$

and the IC

$$
y(0, x)=y_{0}(x), \quad y_{t}(x, 0)=y_{1}(x) .
$$

Here the functions $g^{j}(x) f_{j}(t), j=1,2$ are two independent exterior forces with the given distributions $g^{j}(x)$ along the ring and unknown controls $f_{j}(t)$; the factor $\rho$ in the right-hand-side is introduced for convenience.

We will need the space

$$
H_{p e r}^{1}(0, l):=\left\{\phi \in H^{1}(0, l): \phi(0)=\phi(l)\right\} .
$$

\section{Assumptions}

1. $\rho>0 ; \quad P(t) \in C^{2}[0, T]$ and strictly positive.

2. $y_{k}(x), k=0,1$ and $g^{j}(x), f_{j}(t), j=1,2$ are real-valued.

3. $g^{j}(x) \in L^{2}(0, l) ; f_{j}(t) \in L^{2}(0, T), j=1,2$.

4. $y_{0}(x) \in H_{p e r}^{1}(0, l), y_{1}(x) \in L^{2}(0, l)$.

It is well-known ([19]) that IBVP above has a unique solution if the functions $y_{0}, y_{1}, g^{j}$, and $f_{j}$ are smooth enough. Our goal is to find the conditions on the system under consideration that guarantee the existence of controls $f_{j}(t)$ such that the ring goes to rest in a given time $T$ and construct these controls.

A ring being a manifold without boundary, boundary control does not make sense in this case and we have to use distributed controls.

It appears (see [4]) that for a ring, one control is not enough for controllability; that is why there are two terms in the right-hand-side of (5.1).

\subsection{The main results}

The eigenfunctions associated with the homogeneous PDE (5.1) subject to the periodic BC are the solutions of the SLP,

$$
e^{\prime \prime}(x)+\lambda \rho e(x)=0, \quad 0<x<l, e(0)=e(l), e^{\prime}(0)=e^{\prime}(l) .
$$

It is well-known (even for variable $\rho$, see, e.g. [20]) that the eigenfunctions $\left\{e_{n}(x), n \in \mathbb{Z}\right\}$ form an orthogonal basis in $L_{\rho}^{2}(0, l)$; we always may assume it to be orthonormal. For constant $\rho$, the 
multiplicity of the eigenvalues $\lambda_{n}, n \neq 0$ is two. Actually the multiplicity of the eigenvalues is the mathematical reason why in general two controls are required to control the ring. In turn, the presence of two controls reduces the interval of controllability to the optical length of the ring comparing with the double optical length in the case of one control as in Sections 1-4.

The following result is obvious.

Lemma 10. The eigenfunctions and eigenvalues of SLP (5.5) have the following form,

$$
\begin{gathered}
e_{n}(x)=\sqrt{\frac{2}{\rho l}} \cos \frac{2 \pi n x}{l}, e_{-n}(x)=\sqrt{\frac{2}{\rho l}} \sin \frac{2 \pi n x}{l}, n \in \mathbb{N} ; \quad e_{0}(x)=\sqrt{\frac{1}{\rho l}}, \\
\lambda_{ \pm n}=\left(\frac{2 \pi n}{l \sqrt{\rho}}\right)^{2}=\left(\frac{2 \pi n}{\mathbf{L}}\right)^{2} .
\end{gathered}
$$

The existence, uniqueness and regularity of the solution for IBVP are described by the following

Theorem 3. Let Assumptions 1-4 be satisfied. Then, there exists a unique solution for IBVP (5.1)(5.3) satisfying the following inclusions,

$$
y \in C\left([0, T] ; H_{p e r}^{1}(0, l)\right), y_{t} \in C\left([0, T] ; L^{2}(0, l)\right) .
$$

$l$-periodicity of the problem and Assumptions 3-4 for the initial data $y_{k}(x)$ and the space distributions of the controls $g^{j}(x)$ allow representing them as the Fourier series with respect to the basis $\left\{e_{n}(x), n \in \mathbb{Z}\right\}$ with the (known) coefficients $y_{n}^{j}$ and $g_{n}^{j}$ correspondingly,

$$
\begin{aligned}
& y_{k}(x)=\sum y_{n}^{k} e_{n}(x), k=0,1, \\
& g^{j}(x)=\sum g_{n}^{j} e_{n}(x), j=1,2 .
\end{aligned}
$$

Here and below the absence of the summation index means that it is carried over all $n \in \mathbb{Z}$. Assumption 3 implies

$$
\left\|g^{j}\right\|^{2}=\sum\left|g_{n}^{j}\right|^{2}<\infty
$$

We recall the notation:

$$
\mathbb{Z}^{\prime}=\mathbb{Z} \backslash\{0\}
$$

We proceed further under the following assumptions on the functions $g^{j}(x)$.

Assumption 5.

$$
\begin{array}{ll}
\text { 5a. } & \triangle_{n} \equiv g_{n}^{1} g_{-n}^{2}-g_{n}^{2} g_{-n}^{1} \neq 0 \quad \forall n \in \mathbb{Z}^{\prime} . \\
\text { 5b. } & \left|g_{0}^{1}\right|+\left|g_{0}^{2}\right| \neq 0 . \diamond
\end{array}
$$

We note that the identity $\triangle_{-n}=-\triangle_{n}, n \in \mathbb{Z}^{\prime}$ holds. 
The meaning of the assumption is that we actually consider controllability of the system in the "general position", as it is rather common in Physics.

The specific examples considered in [4] demonstrate that. Specifically, the space distribution of the controls there has the form of the Dirac delta (or its derivative) at two points $x_{1}, x_{2}$, such that $\left(x_{2}-x_{1}\right) / \pi$ is irrational.

The main result of this section is given by

Theorem 4. Let Assumptions 1-5 be satisfied. If

$$
\sum_{n \in \mathbb{Z}^{\prime}}\left|\frac{g_{-n}^{j} \lambda_{n} y_{n}^{0}}{\triangle_{n}}\right|^{2}<\infty \text { and } \sum_{n \in \mathbb{Z}^{\prime}}\left|\frac{g_{-n}^{j} y_{n}^{1}}{\triangle_{n}}\right|^{2}<\infty, j=1,2,
$$

and $T>T_{0}$, then, there exist the functions $f_{1,2} \in L^{2}(0, T)$, such that

$$
y(x, T)=y_{t}(x, T)=0, \quad x \in(0, l) .
$$

Here $T_{0}$ is the optical length of the ring defined by the same equation as the optical length of the string (see (2.20)):

$$
\int_{0}^{T_{0}} \sqrt{P(t)} d t=l \sqrt{\rho}=\mathbf{L} .
$$

We note that the inequalities (5.14) represent some restrictions on both initial data and space distributions of the controls.

Theorem 4 implies that the system (5.1), (5.2) is spectrally controllable. This means that the set of initial data $\left\{y_{0}, y_{1}\right\}$ which can be steered to zero at time $T$ (see (5.15)) contains $E \times E$, where $E$ is the set of all finite linear combinations of the eigenfunctions $e_{n}$.

Spectral controllability follows from exact controllability and implies approximate controllability; spectral controllability is important for many problems of control theory and its applications (see, e.g. [28]) as well as for solving inverse problems for PDEs [13], [7].

The counterpart of Theorem 4 can be formulated as follows.

Theorem 5. The system (5.1), (5.2) is not spectrally controllable for $T \leq T_{0}$.

\subsection{The moment problem}

We construct the solution for IBVP (5.1)-(5.3) in the form of the Fourier series with respect to the basis $\left\{e_{n}(x), n \in \mathbb{Z}\right\}$ with the unknown coefficients $A_{n}(t)$ satisfying the Cauchy problem,

$$
\ddot{A}_{n}+\lambda_{n} P(t) A_{n}=\sum_{j=1}^{2} g_{n}^{j} f_{j}(t), \quad A_{n}(0)=y_{n}^{0}, \dot{A}_{n}(0)=y_{n}^{1}, \quad n \in \mathbb{Z} .
$$

It is natural to rewrite the ODEs (5.17) using the notation (2.23),

$$
\ddot{A}_{n}+\omega^{2}(t) \lambda_{n} A_{n}=\sum_{j=1}^{2} g_{n}^{j} f_{j}(t), \quad n \in \mathbb{Z}
$$


We introduce the Liouville transformation (2.23) exactly as we did it for the string problem. We also introduce the new unknown functions according to the formulas

$$
A_{n}(t)=\omega^{-1 / 2}(t) \hat{A}_{n}(s), \quad n \in \mathbb{Z} .
$$

Applying the transformation (2.23), (5.19) to the ODEs and initial conditions (5.17)-(5.18) yields a new Cauchy problem,

$$
\begin{gathered}
\hat{A}_{n}^{\prime \prime}+\left(Q(s)+\lambda_{n}\right) \hat{A}_{n}=\sum_{j=1}^{2} g_{n}^{j} \hat{f}_{j}(s), \\
\hat{A}_{n}(0)=a_{n}^{0}, \hat{A}_{n}^{\prime}(0)=a_{n}^{1}, \quad n \in \mathbb{Z}, \\
\text { where } a_{n}^{0}=\omega^{1 / 2}(0) y_{n}^{0}, \quad a_{n}^{1}=\omega^{-1 / 2}(0) y_{n}^{1}+\frac{1}{2} \omega^{-3 / 2}(0) \dot{\omega}(0) y_{n}^{0} .
\end{gathered}
$$

The following functions are introduced here,

$$
Q(s)=\frac{3 \dot{\omega}(t)^{2}}{4 \omega^{4}(t)}-\frac{\ddot{\omega}(t)}{2 \omega^{3}(t)} \text { and } \hat{f}_{j}(s)=\frac{f_{j}(t)}{\omega(t)^{3 / 2}} \text { with } t=t(s) .
$$

It is helpful to note at this moment that the conditions (5.14) are equivalent to the following,

$$
\sum_{n \in \mathbb{Z}^{\prime}}\left|\frac{g_{-n}^{j} \lambda_{n} a_{n}^{0}}{\triangle_{n}}\right|^{2}<\infty \text { and } \sum_{n \in \mathbb{Z}^{\prime}}\left|\frac{g_{-n}^{j} a_{n}^{1}}{\triangle_{n}}\right|^{2}<\infty, j=1,2 .
$$

The solution to the Cauchy problem (5.20) may be given in terms of two independent solutions of the corresponding homogeneous ODE,

$$
w_{n}^{\prime \prime}+\left(Q(s)+\lambda_{n}\right) w_{n}=0 \quad n=0,1,2, \ldots
$$

We introduce two linearly independent solutions $\Phi_{n}(s)$ and $\Psi_{n}(s)$ of (5.24) satisfying different initial conditions,

$$
\begin{gathered}
\Phi_{0}^{\prime \prime}+Q(s) \Phi_{0}=0, \quad \Phi_{0}(0)=0, \quad \Phi_{0}^{\prime}(0)=1, \\
\Psi_{0}^{\prime \prime}+Q(s) \Psi_{0}=0, \quad \Psi_{0}(0)=1, \quad \Psi_{0}^{\prime}(0)=0, \\
\Phi_{n}^{\prime \prime}+\left(Q(s)+\lambda_{n}\right) \Phi_{n}=0, \quad \Phi_{n}(0)=0, \quad \Phi_{n}^{\prime}(0)=\sqrt{\lambda_{n}}, \quad n \in \mathbb{N}, \\
\Psi_{n}^{\prime \prime}+\left(Q(s)+\lambda_{n}\right) \Psi_{n}=0, \quad \Psi_{n}(0)=1, \quad \Psi_{n}^{\prime}(0)=0, \quad n \in \mathbb{N} .
\end{gathered}
$$

We also introduce a family of functions that will be used below,

$$
\begin{gathered}
\mathcal{F}=\left\{\mathcal{F}_{n}\right\}_{n \geq-1}, \text { where } \\
\mathcal{F}_{2 n}(t)=\Psi_{n}(s), \mathcal{F}_{2 n-1}(s)=\Phi_{n}(s), n \in \mathbb{N} \cup\{0\} .
\end{gathered}
$$

If the tension $P(t)$ is independent on time, the Cauchy problems (5.25)-(5.28) produce a simplified family,

$$
\begin{gathered}
\mathcal{F}^{0}=\left\{\mathcal{F}_{n}^{0}\right\}_{n \geq-1}, \text { where } \\
\mathcal{F}_{-1}^{0}=s, \mathcal{F}_{0}^{0}=1, \mathcal{F}_{2 n}^{0}(s)=\cos \sqrt{\lambda_{n}} s, \mathcal{F}_{2 n-1}^{0}(s)=\sin \sqrt{\lambda_{n}} s, n \geq 1 .
\end{gathered}
$$


Remark 4. Different structure of the spectrum of the SLP (5.5) for $n=0$ and $n \neq 0$ forces us here and below considering the corresponding equations for $n=0$ and $n \neq 0$ separately.

The representation for the (unique) solution to the problem (5.20) and its derivative has now the form,

$$
\begin{aligned}
& \left(\begin{array}{c}
\hat{A}_{0}(s) \\
\hat{A}_{0}^{\prime}(s)
\end{array}\right)=a_{0}^{1}\left(\begin{array}{c}
\Phi_{0}(s) \\
\Phi_{0}^{\prime}(s)
\end{array}\right)+a_{0}^{0}\left(\begin{array}{c}
\Psi_{0}(s) \\
\Psi_{0}^{\prime}(s)
\end{array}\right) \\
& +\int_{0}^{s}\left(\Psi_{0}(\xi)\left(\begin{array}{c}
\Phi_{0}(s) \\
\Phi_{0}^{\prime}(s)
\end{array}\right)-\Phi_{0}(\xi)\left(\begin{array}{l}
\Psi_{0}(s) \\
\Psi_{0}^{\prime}(s)
\end{array}\right)\right) \sum_{j=1}^{2} g_{0}^{j} \hat{f}_{j}(\xi) d \xi \\
& \left(\begin{array}{c}
\hat{A}_{n}(s) \\
\hat{A}_{n}^{\prime}(s)
\end{array}\right)=\frac{a_{n}^{1}}{\sqrt{\lambda_{n}}}\left(\begin{array}{c}
\Phi_{|n|}(s) \\
\Phi_{|n|}^{\prime}(s)
\end{array}\right)+a_{n}^{0}\left(\begin{array}{c}
\Psi_{|n|}(s) \\
\Psi_{|n|}^{\prime}(s)
\end{array}\right) \\
& +\int_{0}^{s}\left(\Psi_{|n|}(\xi)\left(\begin{array}{c}
\Phi_{|n|}(s) \\
\Phi_{|n|}^{\prime}(s)
\end{array}\right)-\Phi_{|n|}(\xi)\left(\begin{array}{c}
\Psi_{|n|}(s) \\
\Psi_{|n|}^{\prime}(s)
\end{array}\right)\right) \sum_{j=1}^{2} \frac{g_{n}^{j}}{\sqrt{\lambda_{n}}} \hat{f}_{j}(\xi) d \xi, \quad n \in \mathbb{Z}^{\prime} .
\end{aligned}
$$

We are looking for the exterior controls $f_{j}(t)$ (or $\hat{f}_{j}(s)$ ) that drive the solution $y(x, t)$ to rest in the given time $T$, i.e.

$$
A_{n}(T)=\dot{A}_{n}(T)=0, \quad n \in \mathbb{Z}
$$

According to Liouville transformation (2.23), the value of the variable $s$ that corresponds to $t=T$ is given by $\int_{0}^{T} \omega(\xi) d \xi:=\tau$. Hence, the requirements (5.33), in terms of the transformation (5.19) lead to the conditions

$$
\hat{A}_{n}(\tau)=\hat{A}_{n}^{\prime}(\tau)=0, \quad n \in \mathbb{Z} .
$$

Substituting $s=\tau$ into (5.31)-(5.32) yields the following equations for the controls $\hat{f}_{j}(s)$ that guarantee the exact controllability of the ring,

$$
\begin{gathered}
\int_{0}^{\tau}\left(\Psi_{0}(\xi)\left(\begin{array}{c}
\Phi_{0}(\tau) \\
\Phi_{0}^{\prime}(\tau)
\end{array}\right)-\Phi_{0}(\xi)\left(\begin{array}{c}
\Psi_{0}(\tau) \\
\Psi_{0}^{\prime}(\tau)
\end{array}\right)\right) \sum_{j=1}^{2} g_{0}^{j} \hat{f}_{j}(\xi) d \xi \\
=-a_{0}^{1}\left(\begin{array}{c}
\Phi_{0}(\tau) \\
\Phi_{0}^{\prime}(\tau)
\end{array}\right)-a_{0}^{0}\left(\begin{array}{l}
\Psi_{0}(\tau) \\
\Psi_{0}^{\prime}(\tau)
\end{array}\right), \\
\int_{0}^{\tau}\left(\Psi_{|n|}(\xi)\left(\begin{array}{c}
\Phi_{|n|}(\tau) \\
\Phi_{|n|}^{\prime}(\tau)
\end{array}\right)-\Phi_{|n|}(\xi)\left(\begin{array}{l}
\Psi_{|n|}(\tau) \\
\Psi_{|n|}^{\prime}(\tau)
\end{array}\right)\right) \sum_{j=1}^{2} \frac{g_{n}^{j}}{\sqrt{\lambda_{n}}} \hat{f}_{j}(\xi) d \xi \\
=-\frac{a_{n}^{1}}{\sqrt{\lambda_{n}}}\left(\begin{array}{c}
\Phi_{|n|}(\tau) \\
\Phi_{|n|}^{\prime}(\tau)
\end{array}\right)-a_{n}^{0}\left(\begin{array}{c}
\Psi_{|n|}(\tau) \\
\Psi_{|n|}^{\prime}(\tau)
\end{array}\right), n \in \mathbb{Z}^{\prime} .
\end{gathered}
$$


Equalities (5.35)-(5.36) represent a "coupled moment problem" for the unknown controls $\hat{f}_{j}(\xi)$. The problem can be decoupled, and we get separate moment problems for $\hat{f}_{1}(\xi)$ and $\hat{f}_{2}(\xi)$. We now describe this decoupling.

We introduce matrices

$$
\mathcal{W}_{n}=\left(\begin{array}{ll}
\Phi_{|n|}(\tau) & -\Psi_{|n|}(\tau) \\
\Phi_{|n|}^{\prime}(\tau) & -\Psi_{|n|}^{\prime}(\tau)
\end{array}\right), \quad n \in \mathbb{N} \cup\{0\}
$$

the (unknown) vectors

$$
\overrightarrow{\mathcal{X}}_{n}=\int_{0}^{\tau}\left(\begin{array}{l}
\Psi_{|n|}(\xi) \\
\Phi_{|n|}(\xi)
\end{array}\right) \sum_{j=1}^{2} g_{n}^{j} \hat{f}_{j}(\xi) d \xi, \quad n \in \mathbb{Z}
$$

and the (known) vectors

$$
\overrightarrow{\mathcal{A}_{0}}=\left(\begin{array}{c}
-a_{0}^{1} \\
a_{0}^{0}
\end{array}\right) ; \quad \overrightarrow{\mathcal{A}_{n}}=\left(\begin{array}{c}
-a_{n}^{1} \\
|n| a_{n}^{0}
\end{array}\right), \quad n \in \mathbb{Z}^{\prime} .
$$

Using the notations (5.37)-(5.39) in the systems (5.35)-(5.36), we get

$$
\mathcal{W}_{n} \overrightarrow{\mathcal{X}}_{n}=\mathcal{W}_{n} \overrightarrow{\mathcal{A}}_{n}, \quad n \in \mathbb{Z}
$$

The matrices $\mathcal{W}_{n}$ are invertible because of the linear independence of $\Phi_{n}$ and $\Psi_{n}$ for all $n \in$ $\mathbb{N} \cup\{0\}$. Hence, the linear algebraic systems (5.40) have the unique solution,

$$
\overrightarrow{\mathcal{X}}_{n}=\overrightarrow{\mathcal{A}}_{n}, \quad n \in \mathbb{Z}
$$

Rewriting (5.41) in terms of (5.38) and (5.39), we get (not yet separated) moment problems for the controls $\hat{f}_{j}(s)$,

$$
\begin{gathered}
\int_{0}^{\tau}\left(\begin{array}{l}
\Psi_{0}(\xi) \\
\Phi_{0}(\xi)
\end{array}\right) \sum_{j=1}^{2} g_{0}^{j} \hat{f}_{j}(\xi) d \xi=\left(\begin{array}{c}
-a_{0}^{1} \\
a_{0}^{0}
\end{array}\right) \\
\int_{0}^{\tau}\left(\begin{array}{l}
\Psi_{|n|}(\xi) \\
\Phi_{|n|}(\xi)
\end{array}\right) \sum_{j=1}^{2} g_{n}^{j} \hat{f}_{j}(\xi) d \xi=\left(\begin{array}{c}
-a_{n}^{1} \\
|n| a_{n}^{0}
\end{array}\right), \quad n \in \mathbb{Z}^{\prime} .
\end{gathered}
$$

First, we work with Equations (5.43) and rewrite them in another form, for $n \in \mathbb{N}$ only,

$$
\int_{0}^{\tau} \mathcal{G}_{n}\left(\begin{array}{c}
\hat{f}^{1}(\xi) \\
\hat{f}^{2}(\xi)
\end{array}\right) \Psi_{n}(\xi) d \xi=\left(\begin{array}{c}
-a_{n}^{1} \\
-a_{-n}^{1}
\end{array}\right), \quad \int_{0}^{\tau} \mathcal{G}_{n}\left(\begin{array}{c}
\hat{f}^{1}(\xi) \\
\hat{f}^{2}(\xi)
\end{array}\right) \Phi_{n}(\xi) d \xi=\left(\begin{array}{c}
n a_{n}^{0} \\
n a_{-n}^{0}
\end{array}\right), \quad n \in \mathbb{N}
$$

where the invertible matrix $\mathcal{G}_{n}$ is used

$$
\mathcal{G}_{n}=\left(\begin{array}{cc}
g_{n}^{1} & g_{n}^{2} \\
g_{-n}^{1} & g_{-n}^{2}
\end{array}\right), n \in \mathbb{N}
$$


Introducing the moments of control functions,

$$
C_{n}^{j}:=\int_{0}^{\tau} \Psi_{n}(\xi) \hat{f}_{j}(\xi) d \xi, \quad S_{n}^{j}:=\int_{0}^{\tau} \Phi_{n}(\xi) \hat{f}_{j}(\xi) d \xi, \quad n \in \mathbb{N}, j=1,2,
$$

we get, instead of (5.44), the system of equations for the moments,

$$
\left(\begin{array}{c}
C_{n}^{1} \\
C_{n}^{2}
\end{array}\right)=\mathcal{G}_{n}^{-1}\left(\begin{array}{c}
-a_{n}^{1} \\
-a_{-n}^{1}
\end{array}\right), \quad\left(\begin{array}{c}
S_{n}^{1} \\
S_{n}^{2}
\end{array}\right)=\mathcal{G}_{n}^{-1}\left(\begin{array}{c}
n a_{n}^{0} \\
n a_{-n}^{0}
\end{array}\right), n \in \mathbb{N} .
$$

Assumption $5 a$ allows finding the moments $S_{n}^{j}, C_{n}^{j}, n \in \mathbb{Z}^{\prime}, j=1,2$ uniquely from the systems (5.47) and Assumption $5 b$ allows finding the moments $S_{0}^{j}, C_{0}^{j}$, not uniquely though, from (5.42).

To formulate the final form of the moment problem, we let

$$
\mathcal{F}=\left\{\mathcal{F}_{n}\right\}_{n \geq-1}, \text { where } \mathcal{F}_{2 n}(t)=\Psi_{n}(s), \mathcal{F}_{2 n-1}(s)=\Phi_{n}(s), n \in \mathbb{N} \cup\{0\} .
$$

Then, the final form of two separate moment problems for controls $\hat{f}^{1}$ and $\hat{f}^{2}$ with respect to the system of functions $\mathcal{F}$ in $L^{2}(0, \tau)$ is as follows,

$$
\left(\hat{f}_{j}, \mathcal{F}_{n}\right)=\mathcal{B}_{n}^{j}, \quad n \in \mathbb{N} \cup\{0\} \cup\{-1\}, \quad j=1,2
$$

with the coefficients $\mathcal{B}_{n}^{j}$ found uniquely for $n \in \mathbb{N}$ from (5.47) and not uniquely for $n=0$ from (5.42). Here $(\cdot, \cdot)$ is the inner product in $L^{2}(0, \tau)$. Conditions (5.14) and, equivalently, (5.23) imply $\left\{\mathcal{B}_{n}^{j}\right\} \in l^{2}$. We note that the form (5.49) of the moment problem is equivalent to the form (5.47) because the functions $\Phi_{n}(s), \Psi_{n}(s)$ are real-valued.

We have reduced the problem of controllability to the moment problem for the functions $f_{j}(\xi), j=1,2$. The solvability of the moment problem is based on the basis property of the family $\mathcal{F}$.

\subsection{Controllability}

The next result may be proven with the help of the same methods as in Section 3.

Lemma 11. The families $\mathcal{F}$ and $\mathcal{F}^{0}$ are quadratically close. The family $\mathcal{F}$ forms an $\mathcal{L}$-basis in $L^{2}(0, \tau)$ for any $\tau>\mathbf{L}$.

According to this lemma, the family $\mathcal{F}$ forms an $\mathcal{L}$-basis in $L^{2}(0, \tau)$, i.e. a Riesz basis in the closure of its linear span $\overline{\mathcal{L}}$, for all $\tau>\mathbf{L}$. We fix an arbitrary $\tau>\mathbf{L}$ and introduce a biorthogonal basis $\mathcal{F}^{b}=\left\{\mathcal{F}_{n}^{b}\right\} \subset \overline{\mathcal{L}}$ to the Riesz basis $\mathcal{F}$, so that

$$
\left(\mathcal{F}_{n}, \mathcal{F}_{m}^{b}\right)=\delta_{n}^{m}, \forall n, m \geq-1
$$

The moment equations (5.49) mean that $\left\{\mathcal{B}_{n}^{j}\right\}$ are the Fourier coefficients of $\hat{f}_{j}$ with respect to the basis $\mathcal{F}$. Hence, the functions $\hat{f}_{j}$ may be reconstructed as follows,

$$
\hat{f}_{j}(s)=\sum_{n}\left(\hat{f}_{j}, \mathcal{F}_{n}\right) \mathcal{F}_{n}^{b}(s)=\sum_{n} \mathcal{B}_{n}^{j} \mathcal{F}_{n}^{b}(s)
$$


This reconstruction is not unique because an arbitrary element from the orthogonal complement to $\overline{\mathcal{L}}$ may be added to $\hat{f}_{j}(s)$ and the dimension of that complement is infinite (see, e.g. [5, Sec. II.4.6], [8]).

This proves Theorem 4 which states a positive result on controllability of the system (5.1), (5.2) for $T>T_{0}$.

Since the family $\mathcal{F}$ is quadratically close to $\mathcal{F}^{0}$, it can be shown exactly as in the proof of Theorem 2 that, for any $\tau \leq \mathbf{L}$, there exist a proper subfamily of $\mathcal{F}$ which forms a Riesz basis in $L^{2}(0, \tau)$. Therefore, $\mathcal{F}$ is not minimal in $L^{2}(0, \tau)$ for $\tau \leq \mathbf{L}$, and the system (5.1), (5.2) is not spectrally controllable in the time interval $[0, T]$ if $T \leq T_{0}$ (see [5, Sec. III.3]). Theorem 5 is proved.

Remark 5. In contrast to the case of boundary control considered in Sections 1-4, the system (5.1), (5.2) can be approximately controllable in arbitrary small time interval $[0, T]$ if the Fourier coefficients $g_{n}^{j}$ are not zero and decrease sufficiently fast as $n \rightarrow \pm \infty$ (see [5, Sections II.6.4 and III.3]). Certainly, this controllability is not stable (non-robust) with respect to small perturbations of the system parameters.

\section{Acknowledgments}

This work was started in March 2009 while S. A. Avdonin visited the Dipartimento di Matematica, Politecnico di Torino, as a visiting professor supported by GNAMPA-INDAM. S. A. Avdonin is grateful to the Dipartimento di Matematica for its hospitality.

S. A. Avdonin's research was supported in part by the National Science Foundation, grant ARC 0724860.

B. P. Belinskiy's research was supported in part by a University of Tennessee at Chattanooga Faculty Research Grant.

L. Pandolfi's research fits the research programs of GNAMPA-INDAM, in the framework of the research proposed for the "Groupement de Recherche en Contrôle des EDP entre la France et IItalie (CONEDP-CNRS)".

The authors are grateful to Don Hinton for useful discussion and to anonymous referee for helpful remarks.

\section{References}

[1] S. A. Avdonin and B. P. Belinskiy, Controllability of a string under tension. Discrete and Continuous Dynamical Systems: A Supplement Volume, (2003), 57-67.

[2] S. A. Avdonin and B. P. Belinskiy, On the basis properties of the functions arising in the boundary control problem of a string with a variable tension. Discrete and Continuous Dynamical Systems: A Supplement Volume, (2005), 40-49. 
[3] S. A. Avdonin and B. P. Belinskiy, On controllability of a rotating string. J. Math. Anal. Appl., 321 (2006), 198-212.

[4] S. A. Avdonin, B. P. Belinskiy, and S. A. Ivanov, On controllability of an elastic ring. Appl. Math. Optim., 60 (2009), 71-103.

[5] S. A. Avdonin and S. A. Ivanov. Families of Exponentials. The Method of Moments in Controllability Problems for Distributed Parameter Systems. Cambridge University Press, New York, 1995.

[6] S. A. Avdonin and S. A. Ivanov, Exponential Riesz bases of subspaces and divided differences. St. Petersburg Mathematical Journal, 13 (2001), 339-351.

[7] S. Avdonin, S. Lenhart, and V. Protopopescu, Solving the dynamical inverse problem for the Schrödinger equation by the Boundary Control method. Inverse Problems, 18 (2002), 41-57.

[8] S. Avdonin and W. Moran, Ingham type inequalities and Riesz bases of divided differences. Int. J. Appl. Math. Comput. Sci., 11 (2001), 101-118.

[9] S. A. Avdonin and W. Moran, Simultaneous control problems for systems of elastic strings and beams. Systems and Control Letters, 44 (2001), 147-155.

[10] S. A. Avdonin and M. Tucsnak, On the simultaneously reachable set of two strings. ESAIM: Control, Optimization and Calculus of Variations, 6 (2001), 259-273.

[11] V. Barbu and M. Iannelli, Approximate controllability of the heat equation with memory. Differential and Integral Equations, 13 (2000), 1393-1412.

[12] B. P. Belinskiy, J. P. Dauer, C. F. Martin, and M. A. Shubov, On controllability of an elastic string with a viscous damping. Numerical Functional Anal. and Optimization, 19 (1998), 227-255.

[13] M. I. Belishev, Canonical model of a dynamical system with boundary control in inverse problem for the heat equation. St. Petersburg Math. Journal, 7, (1996), 869-890.

[14] A. Erdélyi. Asymptotic Expansions. Dover Publications, Inc., 1956.

[15] I. C. Gohberg and M. G. Krein. Introduction to the Theory of Linear Nonselfadjoint Operators", Translations of Mathematical Monographs. American Mathematical Society. 18, Providence, RI, 1969.

[16] J. P. Den Hartog. Mechanical Vibrations. McGraw-Hill Book Company, New York, 1956.

[17] S. Hansen and E. Zuazua, Exact controllability and stabilization of a vibrating string with an interior point mass. SIAM J. Control Optim., 33 (1995), 1357-1391.

[18] T. von Kàrmàn and M. A. Biot. Mathematical Methods in Engineering. McGraw-Hill Book Company, New York, 1940. 
[19] O. A. Ladyzhenskaia. The Boundary Value Problems of Mathematical Physics. SpringerVerlag, New York, 1985.

[20] B. M. Levitan and I. S. Sargsjan. Sturm-Liouville and Dirac Operators. Translated from the Russian. Mathematics and its Applications (Soviet Series), 59. Kluwer Academic Publishers Group, Dordrecht, 1991.

[21] N. W. McLachlan. Theory and Applications of Mathieu Functions, Oxford, 1947.

[22] A. V. Metrikine and M. V. Tochilin, Steady-state vibrations of an elastic ring under moving load. J. Sound and Vibration, 232 (2000), 511-524.

[23] L. Pandolfi, The controllability of the Gurtin-Pipkin equation: a cosine operator approach. Applied Mathematics and Optimization, 52 (2005), 143-165.

[24] L. Pandolfi, Riesz system and the controllability of heat equations with memory. Integral Eq. Oper. Theory, 64 (2009), 429-453.

[25] L. Pandolfi, Riesz systems, spectral controllability and an identification problem for heat equations with memory. Quaderni del Dipartimento di Matematica, Politecnico di Torino, "La Matematica e le sue Applicazioni” n. 6-2009 (in print, Discr. Cont. Dynam. Systems).

[26] L. Pandolfi, Riesz systems and moment method in the study of viscoelasticity in one space dimension. Quaderni del Dipartimento di Matematica, Politecnico di Torino, "La Matematica e le sue Applicazioni” n. 5-2009 (in print, Discr. Cont. Dynam. Systems).

[27] D. L. Russell, Nonharmonic Fourier series in the control theory of distributed parameter systems. J. Math. Anal. Appl., 18 (1967), 542-559.

[28] D. L. Russell, Controllability and stabilizability theory for linear partial differential equations. SIAM Review, 20 (1978), 639-739.

[29] D. L. Russell, On exponential bases for the Sobolev spaces over an interval. J. Math.Anal.Appl., 87 (1982), 528-550.

[30] W. Soedel. Vibrations of Shells and Plates. Marcel Dekker, Inc., New York, 1993.

[31] M. E. Taylor. Pseudodifferential Operators. Princeton University Press, Princeton, NJ, 1981.

[32] S. Timoshenko. Thèorie des Vibrations. Libr. Polytecnique Ch Bèranger, Paris, 1947.

[33] V. Z. Vlasov. Obcaya Teoriya Obolocek i e Priloeniya v Tehnike (in Russian) [General Theory of Shells and Its Applications in Technology]. Gosudarstvennoe Izdatel'stvo TehnikoTeoreticeskoi Literatury, Moscow-Leningrad (1949).

[34] X. Fu, J. Yong and X. Zhang, Controllability and observability of the heat equation with hyperbolic memory kernel. J. Diff. Equations, 247 (2009), 2395-2439. 